\title{
High-Speed Imaging Optical Pyrometry for Study of Boron Nitride Nanotube Generation
}

\author{
Jennifer A. Inman, ${ }^{*}$ Paul M. Danehy, ${ }^{\dagger}$ Stephen B. Jones, ${ }^{\dagger}$ and Joseph W. Lee ${ }^{\S}$ \\ NASA Langley Research Center, Hampton, VA, 23681-2199
}

\begin{abstract}
A high-speed imaging optical pyrometry system is designed for making in-situ measurements of boron temperature during the boron nitride nanotube synthesis process. Spectrometer measurements show molten boron emission to be essentially graybody in nature, lacking spectral emission fine structure over the visible range of the electromagnetic spectrum. Camera calibration experiments are performed and compared with theoretical calculations to quantitatively establish the relationship between observed signal intensity and temperature. The one-color pyrometry technique described herein involves measuring temperature based upon the absolute signal intensity observed through a narrowband spectral filter, while the two-color technique uses the ratio of the signals through two spectrally separated filters. The present study calibrated both the one- and two-color techniques at temperatures between $1,173 \mathrm{~K}$ and 1,591 $\mathrm{K}$ using a pco.dimax HD CMOSbased camera along with three such filters having transmission peaks near $550 \mathrm{~nm}, 632.8$ $\mathrm{nm}$, and $800 \mathrm{~nm}$.
\end{abstract}

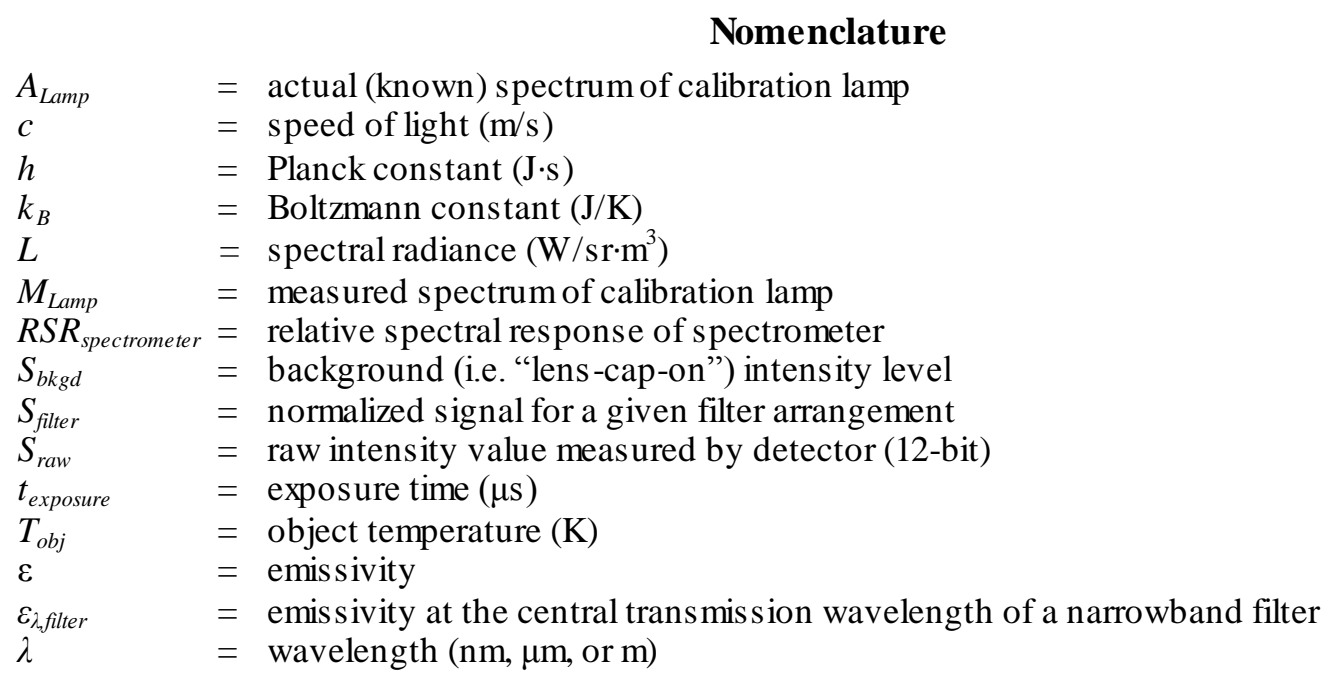

\section{Introduction}

$\mathrm{B}$ ORON nitride nanotubes (BNNTs) and carbon nanotubes (CNTs) are structurally similar and share extraordinary mechanical properties, but they differ in chemical, optical, electrical and thermal properties. For example, BNNTs are electrical insulators while CNTs are conductors. In addition BNNTs have a potential advantage over CNTs for high-temperature applications as they are more resistant to oxidation at high temperatures than CNTs. BNNTs are being produced in a high-pressure reaction chamber at NASA Langley Research Center in Hampton, VA using the pressurized vapor/condenser method. ${ }^{1}$ A $5 \mathrm{~kW} \mathrm{CO}_{2}$ laser is focus ed onto a bundle of boron fibers in a pressurized nitrogen environment. The boron melts, forming a ball that emits boron vapor. The vapor

\footnotetext{
* Research Scientist, Advanced Sensing and Optical Measurement Branch, MS 493, AIAA Member.

${ }^{\dagger}$ Research Scientist, Advanced Sensing and Optical Measurement Branch, MS 493, AIAA Associate Fellow.

${ }^{*}$ Research Technician, Advanced Sensing and Optical Measurement Branch, MS 493.

${ }^{\S}$ Research Technician, Advanced Sensing and Optical Measurement Branch, MS 493.
} 
mixes with the surrounding nitrogen (containing both $\mathrm{N}_{2}$ and $\mathrm{N}$ ) and produces nanotubes. These nanotubes are collected by a collection wire located above the ball. ${ }^{1}$ An image of a molten boron ball attached to the boron fiber bundle during operation and a photograph of the ball after re-solidification is shown in Figure 1.
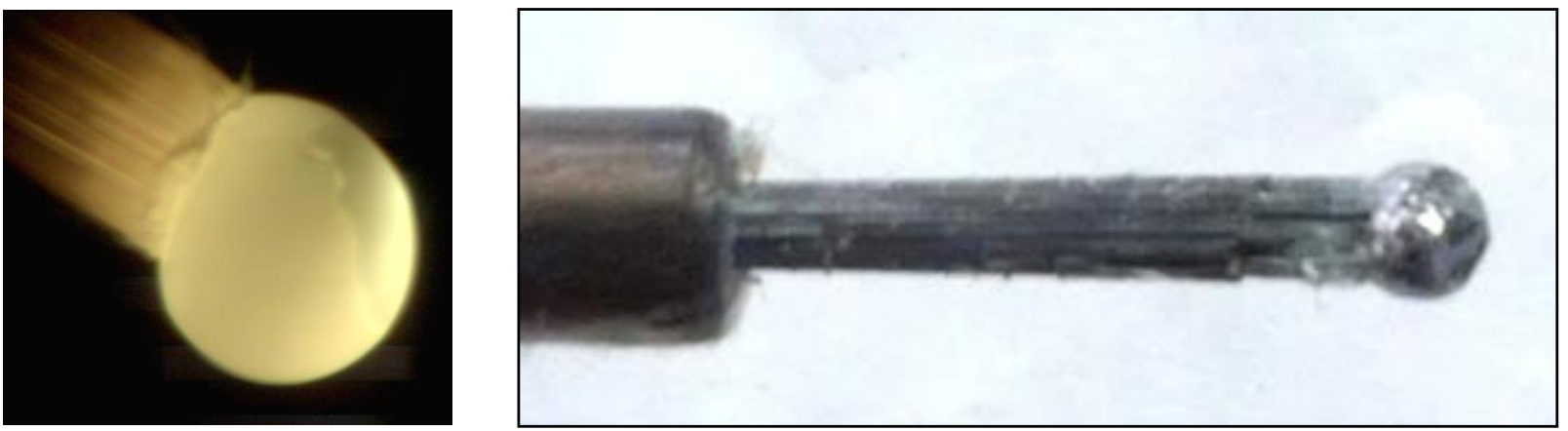

Figure 1. Boron ball attached to the boron fiber bundle. The bundle diameter is about $1 / 8 \mathrm{inch}$. (a) shows an image of the molten ball while it is being heated by a $5 \mathrm{~kW} \mathrm{CO} 2$ laser inside a BNNT production rig; (b) shows the boron ball at room temperature, after re-solidification.

In order to better understand the process of BNNT synthesis, we would like to have a more detailed understanding of how the temperature of the molten boron ball influences the quality and quantity of nanotubes that are produced. Dynamics in the geometry and variations in the temperature of the boron ball are expected on a timescale of microseconds to milliseconds. Therefore, we desire a system capable of providing both high-speed imaging and simultaneous thermal mapping of molten boron inside a BNNT production chamber.

This paper details the design and calibration of such an imaging py rometry system. Spectrometer measurements of molten boron establish that its emis sion across the visible region of the spectrumis ess entially graybody, with an emissivity that is nearly independent of wavelength, simplifying the subsequent camera calibration procedures. These calibration experiments, involving measurements of emission from blackbody calibration sources are described, and the resulting calibration constants are presented.

\section{Theoretical Basics of Optical Pyrometry}

\section{A. Object emission, emissivity, and effect of non-ideal behavior}

Objects at temperatures above absolute zero emit radiation in accordance with Planck's law.

$$
L\left(\lambda, \varepsilon, T_{o b j}\right)=\varepsilon\left(\lambda, T_{o b j}\right) \frac{2 h c^{2}}{\lambda^{5}} \frac{1}{e^{\left(\frac{h c}{\lambda B^{T} o b j}\right)}-1}
$$

$L(\lambda)$ is the spectral radiance at wavelength $\lambda$ (where $\lambda$ has units of meters); $\varepsilon\left(\lambda, T_{o b j}\right)$ is the emissivity at $\lambda$ of an object at temperature $T_{o b j} ; c$ is the speed of light; $h$ is the Planck constant; and $k_{B}$ is the Boltzmann constant. For making measurements in the visible range $(\sim 380-750 \mathrm{~nm})$, this can be simplified using the Wein approximation for temperatures $T \ll h c /\left(k_{B} \lambda\right)(\ll 18,000 \mathrm{~K}$ for $800 \mathrm{~nm}, \ll 22,560 \mathrm{~K}$ for $638.2 \mathrm{~nm}, \ll 26,200 \mathrm{~K}$ for $550 \mathrm{~nm})$. While the temperatures that we haveused in our calibration experiments meet these criteria, we have elected to perform our calculations using the form of Planck's law in Eq. 1 in order to preserve the applicability of our results to the potentially higher temperatures that might be encountered in the BNNT manufacturing process.

\section{B. Spectrometer res ponse curve}

One strategy for making non-imaging temperature measurements at a single point is to record the spectrum of radiation emitted froma given spatial location using a spectrometer and to then fit that measured curve to a library of theoretical emission spectra. For this approach to work well, the emission characteristics of the material in question - namely, its emis sivity as a function of wavelength and temperature, $\varepsilon\left(\lambda, T_{o b j}\right)$ - must be known. For an ideal blackbody, $\varepsilon$ is equal to one (1); for a graybody, $\varepsilon(\lambda)=$ constant (i.e. the emis sivity is independent of wavelength). For real materials, $\varepsilon$ may be a highly variable function of $\lambda$, yielding emission spectra with a great deal of structure. Emis sivity is also generally dependent upon the physical phase (e.g. solid, liquid) of a material, which may in turn be a function of temperature and pressure. 
The relative spectral response ( $R S R$ ) of the spectrometer must be determined prior to obtaining meas urements. This can be accomplished by meas uring the spectrum of a calibration light source whose output spectrum is known. The $R S R$ is determined by dividing the measured spectrum, $M_{\text {Lamp }}$, by the known (actual) spectrum, $A_{\text {Lamp }}$, according to Eq. 2 .

$$
R S R_{\text {spectrometer }}=\frac{M_{\text {Lamp }}}{A_{\text {Lamp }}}
$$

The temperature of an object is then determined by dividing a measured spectrum, $M_{o b j}$, by this $R S R$, to determine the actual emission spectrum of the object, $A_{o b j}$, and then fitting this actual spectrum for temperature. Here, $M_{o b j}$ is understood to be the raw measurement minus any background intensity level, which may depend on both wavelength and the integration time of the detector.

$$
A_{o b j}=\frac{M_{o b j}}{R_{\text {SR }} R_{\text {spectrometer }}}
$$

The $R S R$ of our spectrometer was determined by measuring the emission spectrum of a National Institute of Standards and Technology (NIST) traceable halogen calibration lamp and then dividing that measured spectrum by the known actual emission spectrum of the lamp provided by the manufacturer of the lamp.

\section{Transmission of Optical Filters, Camera Sensor Spectral Responsivity}

In order to add temperature imaging capability rather than measurement at a single point, simultaneous acquisition of the entire s pectrumis exchanged for measurements of absolute light intensity across some (generally narrow) portion of the electromagnetic spectrum. To this end, different high-speed cameras, collection optics and filters were used. In order to calculate the expected signal as a function of temperature, the spectral response of each component between the meas ured object and the camera sensor should be taken into account. Figure 2 shows the product of the manufacturer-measured spectral res ponsivity of the camera and the manufacturer-supplied spectral transmission curves for each of the filters that were used. Calculating the expected signal for a given object temperature through a given filter involved integrating the product of the spectral responsivity of the camera, the spectral transmis sion curve of the filter(s), and the blackbody or graybody emission curve.

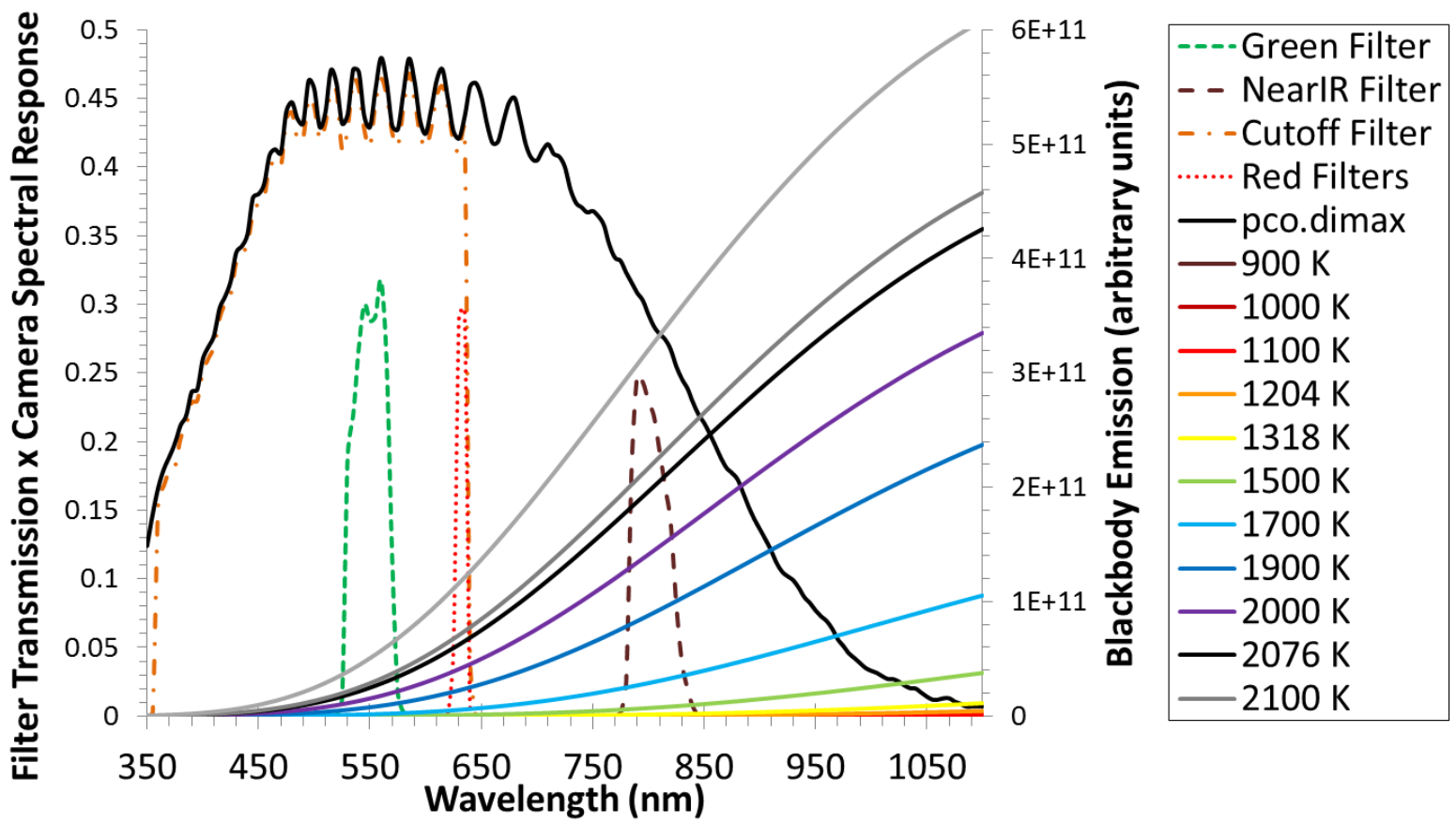

Figure 2. Theoretical graybody emission curves overlaid with spectral transmission curves. The solid black line shows the measured spectral sensitivity of the camera. The dashed lines show the product of this curve with the transmis sion curves of each filter (Green filter $=$ Thorlabs FB550-40; NearIR Filter $=$ Thorlabs FB800-40; Cutoff Filter $=$ Semrock FF01-650/SP; Red Filters $=$ Thorlabs FL05632.8-10 and Semrock FF01-650/SP) 
The camera was a high-speed (1,547 frames per second at full $1920 \times 1080$ pixel resolution) CMOS pco.dimax HD. The four filters used in the present study were a Thorlabs FB550-40 bandpass filter (to be subsequently referred to as the Green filter); a Thorlabs FL05632.8-10 laser line filter used in conjunction with a Semrock FF01-650/SP25 blocking edge BrightLine ${ }^{\circledR}$ short-pass filter (Red filters); and a Thorlabs FB800-40 bandpass filter (Near IR filter). In addition, blackbody curves for the temperatures used in our calibration experiments are shown. In this figure, we have neglected the effect of any spectral variation in the transmission of our camera lens, which we have assumed to be uniformly transparent across the range of our measurements (roughly 500-850 nm). However, the calibrations below should correct for such spectral variations. These particular filters were chosen because their central transmis sion wavelengths coincide with empirical meas urements of the emis sivity of boron that can be found in the literature, ${ }^{2,3}$ as indicated in Table 1 . In addition, one study that employed a broadband measurement of liquid boron emis sivity over the range $450-600 \mathrm{~nm}$ (as opposed to the narrowband, wavelength-s pecific emis sivities given in Table 1) found that the emis sivity decreased almost linearly from 0.35 at $1,490 \mathrm{~K}$ to 0.20 at $2,800 \mathrm{~K}^{4}$

One limitation of ourcalculations is that the spectral response of the camera was only provided up to $1088 \mathrm{~nm}$. While the measured efficiency at this wavelength is less than $1 \%$ and appears to be decreasing, the assumed response - if any - at wavelengths beyond $1,250 \mathrm{~nm}$ could play a significant role in these calculations since the filters themselves have broadband trans mis sion between approximately $10-80 \%$ at longer wavelengths. Because CMOS cameras employ silicon-based sensors, the $\Delta E=1.12 \mathrm{eV}$ optical band gap of silicon (at $300 \mathrm{~K}$; it increases to a theoretical maximum of $1.22 \mathrm{eV}$ at $0 \mathrm{~K}$ ) renders CMOS sensors insensitive to infrared wavelengths with less than this amount of energy. In other words, CMOS sensors cannot absorb photons with wavelengths longer than about $=h c / \Delta E=1.24 \mathrm{eV} \mu \mathrm{m} / 1.12 \mathrm{eV}=1.116 \mu \mathrm{m}^{5,6}$

In choosing to use visible and near-visible wavelengths, the measurements presented herein are negligibly affected by absorption through the air, an effect that must be considered when using different sensors and longer infrared waveleng ths coinciding with absorption features of water. ${ }^{7}$ The use of visible and near-visible wavelengths though, limits the use of the instrument to relatively high temperatures - approximately $900 \mathrm{~K}$ and higher - due to the minimal visible-wavelength emission of objects below these temperatures.

Table 1. Emissivity of boron at the central wavelengths of the filters used in this study.

\begin{tabular}{cccc}
\hline Wavelength & \multicolumn{3}{c}{ Spectral emissivity of boron } \\
\cline { 2 - 4 }$\mu \mathrm{m}$ & Liquid & Smooth solid & Rough solid \\
\hline${ }^{\mathrm{a}} 0.55$ & $0.32 \pm 0.02$ & $0.52 \pm 0.02$ & $0.66 \pm 0.02$ \\
${ }^{\mathrm{b}} 0.6328$ & $0.31 \pm 0.02$ & & 0.68 \\
${ }^{\mathrm{a}} 0.8$ & $0.36 \pm 0.02$ & $0.50 \pm 0.02$ & $0.73 \pm 0.02$ \\
\hline
\end{tabular}

(a) Millot et al. ${ }^{2}$ (b) Krishnan et al. ${ }^{3}$

\section{Experimental Setup}

\section{A. BNNT Science/Production Rig}

Initial measurements with a fiber-optic-coupled Ocean Optics HR2000 spectrometer were performed in the production chamber of a BNNT-s ynthesis rig. A schematic of the layout is shown in Fig. 3. This spectrometer had been used previously for visible emis sion spectroscopy in arc-jet flowfields and was adapted to the current work. ${ }^{8}$ A spherical lens directed light from the test section onto the optical fiber. The spectrometer was set up with nearly 1:1 imaging, using a 200 micron diameter fiber, resulting in a measurement area of slightly less than 200 micron diameter. For alignment, a red (helium neon) laser beam was passed through the fiber, onto the sample, to determine the measurement location. Then the fiber was re-connected to the spectrometer for spectral measurements. An x-y traverse moved the fiber to different locations, which allowed measurements at different spatial locations in the chamber. 


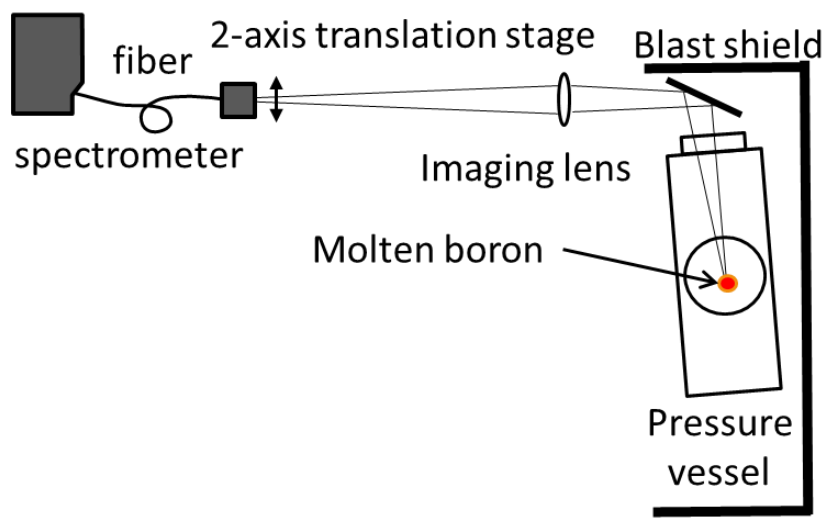

Figure 3. Schematic of spectrometer meas urements in BNNT production chamber. Translation stage allowed lateral and vertical (out-of-the-page) movement of the imaged region. A boron bundle inside a pressure vessel is heated above its melting point by a high-powered laser. The BNNT synthesis rig, including the spectrometer and imaging components, is surrounded by an external enclosure (not shown) for safety, in addition to the blast shield around the pressure vessel.

\section{B. Laboratory Calibration Setup}

In order to calibrate the camera-based measurement system, images were acquired at known temperatures using two NIST-traceable blackbody radiation calibration furnaces: a Mikron M300 and a Mikron M360. The M360 is only capable of producing temperatures up to $1373 \mathrm{~K}\left(1100^{\circ} \mathrm{C}, 2012^{\circ} \mathrm{F}, 2472^{\circ} \mathrm{R}\right)$ but it is portable and comes up to its maximum temperature in aboutan hour. The $\mathrm{M} 300$ is capable of achieving temperatures up to $1591 \mathrm{~K}\left(1318^{\circ} \mathrm{C}\right.$, $2405^{\circ} \mathrm{F}, 2865^{\circ} \mathrm{R}$ ) but requires a forklift or pallet jack to move and requires over 3 hours to come up to its maximum temperature. Each furnace has a small circular aperture providing a view into its hollow spherical interior. A white ceramic material coats the inside of the furnace. The furnaces have stated emissivities of +0.995 for the M $300^{9}$ and $0.999 \pm 0.0005$ for the M360. ${ }^{10}$

Radiation emitted from the furnaces was imaged by a pco.dimax HD camera using a Nikon AF Zoom-Nikkor 35-105 mm f/3.5-4.5 lens focused at $1 \mathrm{~m}$, coinciding with the center of the furnace being imaged. A schematic of the layout is shown in Fig. 4. This resulted in an image considerably smaller than the camera's full $1920 \times 1080$ pixel field of view, so a smaller $480 \times 512$ pixel ROI (region of interest) was used to reduce file size.

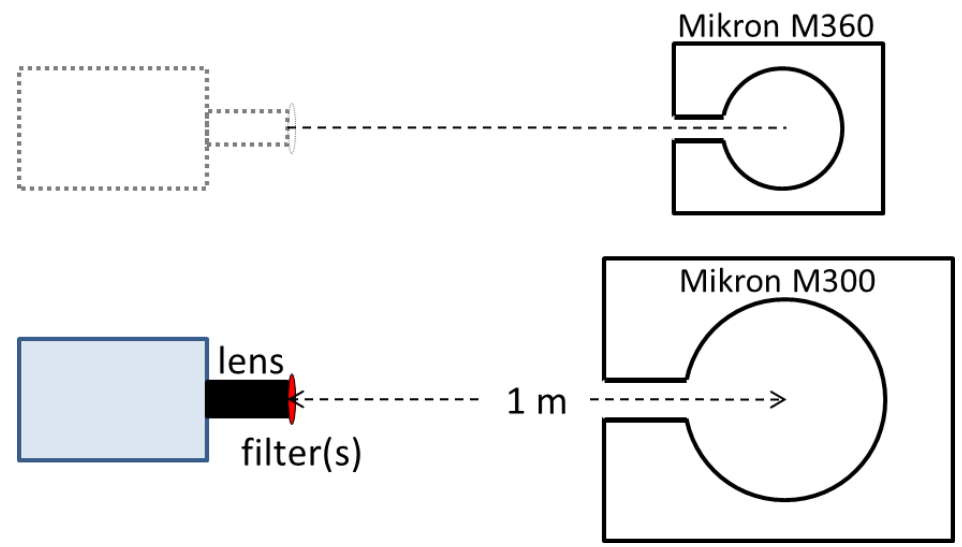

Figure 4. Schematic of setup for camera linearity and calibration measurements. Blackbody radiation calibration sources were imaged through various narrowband filters. This setup was used for both the Phantom 9.0 and pco.dimax studies, although only the M360 was used with the former.

\section{Calibration of Optical Pyrometer}

\section{A. Fitted Temperatures from Spectrometer}

The advantage of using a spectrometer is that an emis sion spectrumcan be obtained, as opposed to a spectrallyintegrated single-intensity. Spectrally-integrated intensity measurements will deviate from graybody behavior if spectral emis sion or absorption lines are present from atoms and/or molecules in the flow. Making measurements 
with a spectrometer allows the wavelength and relative intensity of any such spectral lines to be identified and, if the influence is significant, allows a mitigation strategy to be developed (e.g. using a filter to block emission at particular wavelengths).

Figure 5 shows several spectra obtained on or in the vicinity of the molten boron ball. There were large uncertainties in the precise location of each measurement (the contributions to which are described in more detail below). Presumably, the higher temperatures were measured near the location where the laser impinged on the boron ball while the cooler temperatures were meas ured further away from the impingement point. No spectral emis sion or absorption lines were observed across the measured spectral range at the $0.035 \mathrm{~nm}$ (FWHM) resolution of the spectrometer nor were any wavelength-dependent fine structure features observed. Instead, the observed spectra appeared to exhibit nearly ideal graybody behavior.

Raw spectra havebeen cropped to the region where we had good emis sion from our calibration lamp (350-850 $\mathrm{nm}$ ) and where the spectral response of the spectrometer was relatively large. The spectra had a measured background spectrumsubtracted and were corrected for the relative spectral responsefunction of the spectrometer. They have then been s moothed with a 10-nm wide smoothing (averaging) filter to remove high-frequency noise. Integration times were adjusted to avoid saturation of the detector. Excel's solver was used to perform a least squares fit to graybody curves by varying two free parameters: temperature and amplitude.

A primary disadvantage of this technique is that it is a single point measurement. Consequently, it is time consuming to sample the entire flowfield and/or object and the resulting spatial resolution is low. Surface tension effects, non-uniformheating of the ball, vaporization of the molten boron, and translation of the boron feed stock all contributed further to the spatialuncertainty in the measurement location. These variables made it difficult to say if a given measurement were on the solid or molten part of the boron ball. We did find that the fitted temperatures obtained in the manner just described fell within the expected range, being both above and below the accepted melting point of boron of between 2,350-2,370 K. ${ }^{3,11}$ Nonetheless, these measurements are useful for the following work because they show that the boron radiation does not have significant spectralemis sion or absorption features, suggesting that a lower-spectral-resolution approach would provide reliable temperatures.

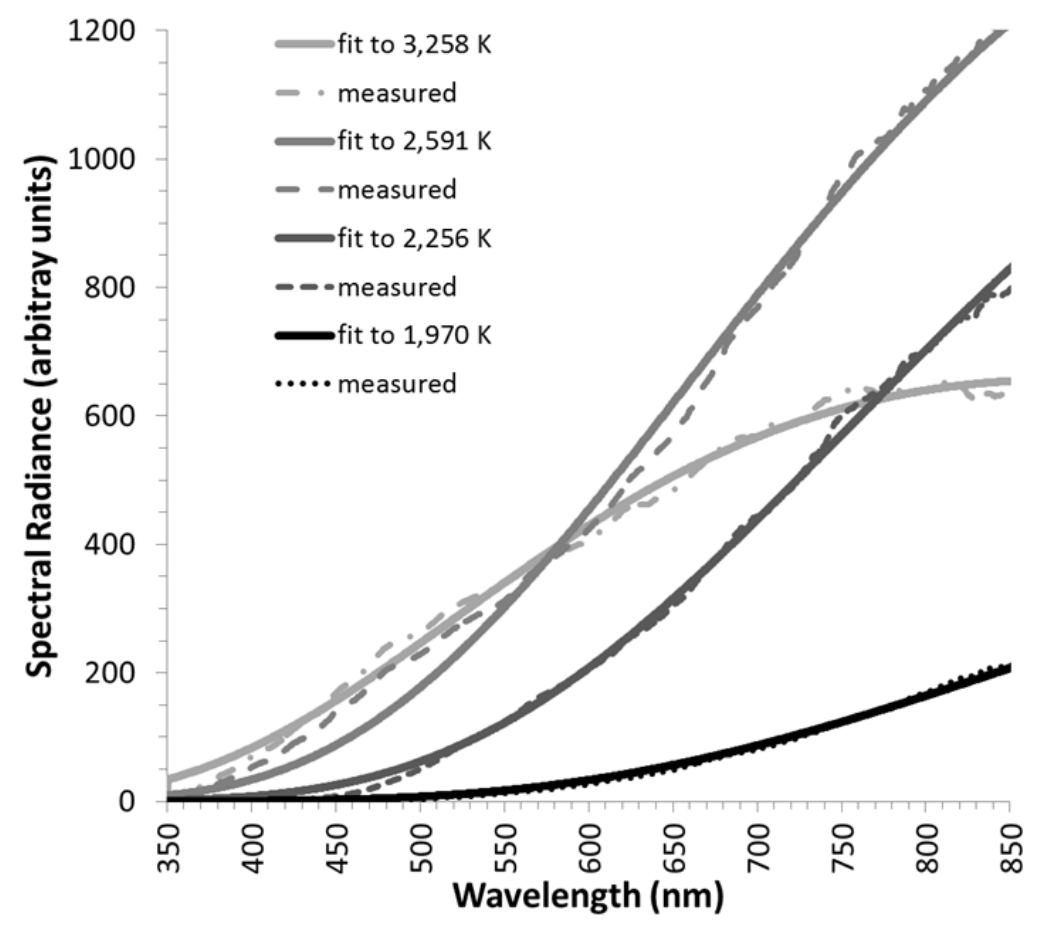

Figure 5. Experimentally measured spectra of boron fitted with theoretical graybody spectra. Spectra were acquired with an Ocean Optics HR2000 spectrometer, were cropped to the spectral region for which we have good calibration data for the relative spectral response of the spectrometer, and have been smoothed by a 10-nm wide averaging filter to remove high-frequency noise. Note that no spectral emis sion lines, absorption features, or other fine structure is observed. 


\section{B. pco.dimax HD Camera Linearity Study}

We first attempted to adapt a Vision Research Phantom 9.1 color high-speed (up to $1000 \mathrm{~Hz}$ ) camera as an optical pyrometer. Phantomcameras are more widely used than the newer and more specialized pco.dimax HD and because this was a color camera, it was hoped that an image-processing algorithmusing the internal color channels of the camera might even allow pyrometry measurements to be obtained without the use of spectral filters. ${ }^{12}$ While others have successfully made temperature measurements with different models of high-speed Phantom cameras, ${ }^{13}$ the camera we tested was found to exhibit non-linearities in its response curve over significant portions of its dynamic range, making it a less attractive candidate for the expected temperature range of our intended application. We instead shifted our efforts to testing a monochrome pco.dimax HD as a camera with a greater bit depth and reportedly better linearity.

In order to determine the linearity of the pco.dimax HD camera's response across its 12-bit dynamic range, a series of measurements were made using the Mikron M360 blackbody calibration source. While the furnace was held at a constant temperature, the exposure time of the camera was varied from its minimum possible value of $1.5 \mu \mathrm{s}$ to its maximum possible value of $40 \mathrm{~ms}$. The mean intensity was calculated over a circular sub-region of the resulting images with an area covering approximately 3,800 pixels. Results are shown in Fig. 6. A linear fit to the resulting data yielded an R-squared value of at least three nines ( 0.999$)$ for intensity levels between $5 \%$ ( 205 counts) and $95 \%$ (3,890 counts) of full-scale (4,095 counts). For intensity levels above this range, the sensor approached saturation and behaved non-linearly. For intensity levels below this range, the effect of the sensor's minimumintensity threshold and sensitivity to the exact background level that was assumed also resulted in non-linear behavior. Other data (not shown in Fig. 6) exposed non-linear effects for exposure times less than $30 \mu s$. Measurements with the lens cap on yielded mean background intensity levels of approximately 43 counts. This mean background level was subtracted from all the raw data prior to further processing.

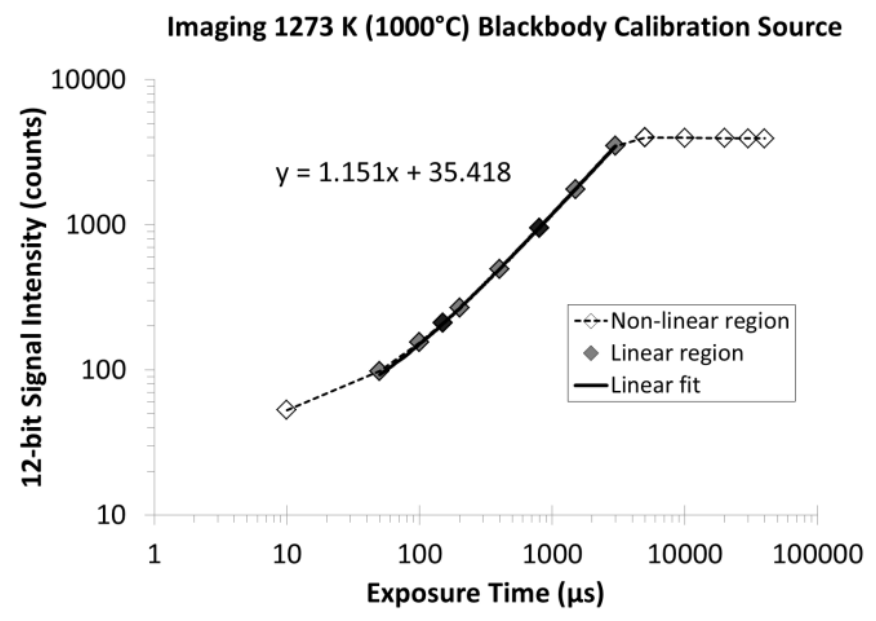

Figure 6. Linearity Test Results for pcodimax CMOS Camera. Intensity versus exposure time is shown on a $\log -\log$ plot for data taken while imaging a blackbody radiation calibration source at $1,273 \mathrm{~K}\left(1000^{\circ} \mathrm{C}\right)$.

\section{Experimental Results}

Two alternative approaches to measuring temperature were considered. The first approach, termed the singlecolortechnique (or one-color pyrometry), attempts to correlate the absolute intensity level measured through a single narrowband filter with a given temperature, since the radiation emitted by an object at a given wavelength is a monotonically increasing function of temperature. The second approach, termed the two-color ratio technique (or two-color pyrometry), uses the relative intensities at two different wavelengths to determine temperature. The approaches and their relative advantages and disadvantages are outlined in the sections below.

\section{A. Single-Color Technique}

The single-color pyrometry technique has the advantage of simplicity. Once the relationship between object temperature and intensity has been establis hed, the image processing procedure is generally relatively quick. If in situ calibration is not an option, however, a calibration point is needed to determine the transfer function between the calibration environment and the experimental environment. The intended application of this technique will involve observations of laser-heated solid and liquid boron at temperatures up to and potentially exceeding 4,000 K. 
The literature indicates that the melting point of boron $(2,350-2,370 \mathrm{~K}$ at atmospheric pressure) is virtually independent of pressure over the range of operating pressures for BNNT synthesis (atmospheric pressure up to 500 psig), increasing at a rate of about $15.5 \mathrm{~K} / \mathrm{GPa}^{11}$ The literature also indicates that boron undergoes an abrupt emissivity change at its melting point (see Table 1). ${ }^{2}$ The boron will pas s through the melting (solid-to-liquid) phase transition on startup and through the solidification (liquid-to-solid) phase transition on shutdown during BNNT synthesis. Thus, there will always be an interface between solid and liquid boron in the camera's field of view during normal operation (see Fig. 1). We anticipate that this phase change (resulting in an emis sivity change) will serve as a calibration point by signaling when the temperature of both the solid and liquid are at the melting point temperature.

Figure 7 show calculations of the expected signal intensity through each filter as a function of temperature, assuming graybody emission. Data points for which the mean raw intensity fell outside the $5 \%-95 \%$ range were eliminated, as were data points for which the exposure time was less than $30 \mu \mathrm{s}$. A background intensity level was subtracted from raw experimentally measured intensity values, and those values were then normalized by the exposure time. The resulting signal that was measured in this manner for a given filter (or filter combination) is labeled $S$, according to Eq. 4 .

$$
S_{\text {filter }}=\frac{S_{\text {raw }}-S_{\text {bkgd }}}{\varepsilon_{\lambda, \text { filter } t_{\text {exposure }}}}
$$

These meas ured signals were multiplied by a single calibration constant and are shown on the same axes as the theoretical curves in Fig. 7. (Note that the data for the Near IR filter is shown with a different vertical axis.) This constant was determined by performing a least squares fit between the calculated values and all of the values measured using the M300 blackbody calibration source.

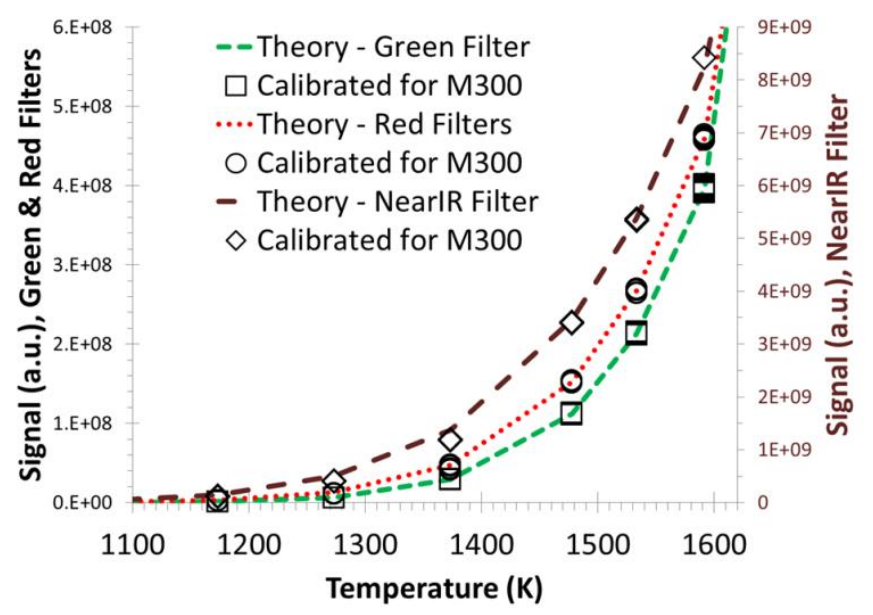

Figure 7. One-Color Pyrometry: Calibrated signal intensity, compared with theory, for three filter arrangements. Note that because the absolute signal level measured with the Near IR filter is so much larger than the signal measured with the other two filter sets, it has been graphed on a secondary vertical axis.

It was found that the calibration constant for all the data taken with the M360 differed slightly from that of the M300. Both furnaces were slightly past their calibration dates and so these calibration constants may need to be adjusted slightly after re-calibrating the furnaces thems elves. Graphing these same data on a logarithmic scale as a function of inverse temperature yields a straight line, as shown in Fig. 8. The solid lines represent the theoretical results (i.e. calculated blackbody curves multiplied by the respective digitized filter function and camera spectral response curve) while the dashed lines indicate the fit to the experimental results. This provides a simple formula, as given in Eq. 5, for extrapolating to the higher temperatures we anticipate encountering in the BNNT generation process. (This equation can be approximately derived from the Planck equation, Eq. 1, for temperatures which render the exponential termin the denominator much greater than one such that the minus one in the denominator can be neglected.) Note though, that these extrapolations can be anchored by another calibration point, as described previously.

$$
\ln \left(S_{\text {filter }}\right)=\frac{a_{\text {filter }}}{T_{\text {obj }}}+b_{\text {filter }}
$$


Here, $a_{\text {filter }}$ is the fitted slope of the lines in Fig. 8 and $b_{\text {filter }}$ is the fitted intercept minus the natural logarithm of the multiplicative constant used to scale the arbitrary intensity units of the detector to the theoretical units. The calibration constants for Eq. 5 for each of our three filter combinations are listed in Table 2. The resulting meas ured temperatures versus setpoint temperatures are shown in Fig. 9. These are the same data points as those shown in Fig. 8, but where Fig. 8 shows how to extract a measured temperature from a given measured signal, Fig. 9 shows the variation in the temperature value calculated using the constants in Table 2 as compared with the setpoint temperature of the calibration source. Since the calibration was performed by performing a least-squares fit to all the M300 data points (in general, the higher temperatures), theindividual data points taken with the M300 show s maller deviations fromthe line of perfect agreement than do those data points taken with the M360 (in general, the lower temperatures).

Rearranging Eq. 5 gives an expression in Eq. 6 for calculating the unknown temperature of a blackbody object (or graybody object, if the emissivity is known) using these calibration constants and the measured normalized intensity with a given filter:

$$
T_{o b j}=\frac{a_{\text {filter }}}{\ln \left(S_{\text {filter }}\right)-b_{\text {filter }}}
$$

If the emis sivity of the measured object is unknown, this changes the second calibration constant and so a calibration point is needed at a known temperature. The second calibration constant can then be recalculated according to Eq. 7 :

$$
b_{\text {filter }}=\ln \left(S_{\text {filter }}\right)-\frac{a_{\text {filter }}}{T_{\text {known }}}
$$

Table 2. One-Color Pyrometry Calibration Constants.

\begin{tabular}{cccc} 
& & \multicolumn{2}{c}{$\begin{array}{c}\text { 1-color pyrometry } \\
\text { constants }\end{array}$} \\
\cline { 3 - 4 } Filter(s) & Filter Part Number & $a$ & $b$ \\
\hline NearlR & Thorlabs FB800-40 & $-19,122$ & 16.726 \\
& & & \\
Red & $\begin{array}{c}\text { Thorlabs FL05632.8-10+ } \\
\text { Semrock FF01-650/SP }\end{array}$ & $-23,591$ & 16.585 \\
& $\begin{array}{c}\text { Thorlabs FB550-40 } \\
\text { Green }\end{array}$ & Ther & \\
\hline
\end{tabular}

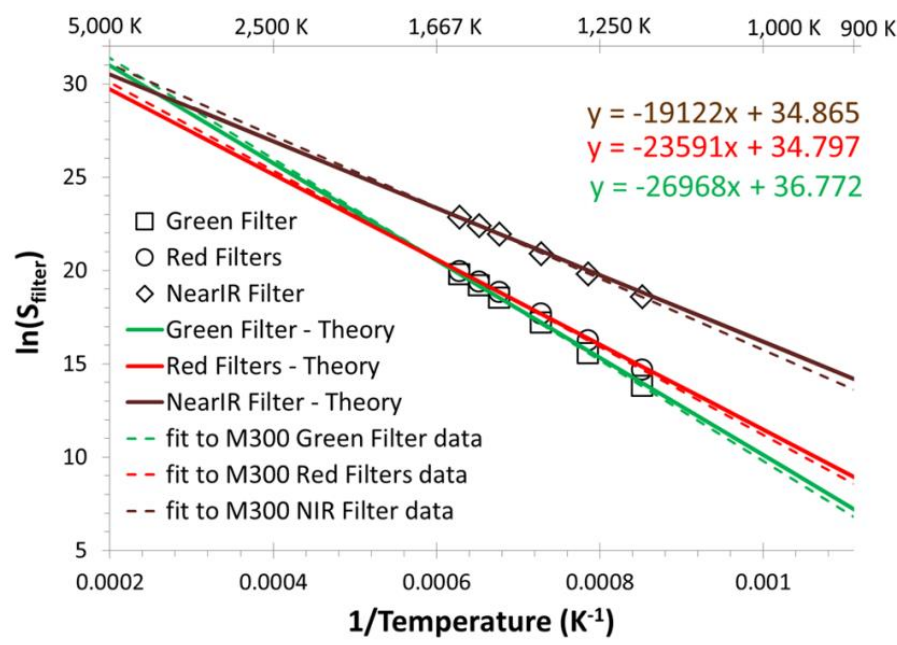

Figure 8. One-Color Pyrometry, $\ln ($ Signal) vs. 1/Temperature, after calibration. Solid lines represent the theoretical curves while dashed lines show the curves fitted to the data taken with the M300 Blackbody Radiation Calibration Source. Equations for the fits are shown, where $y$ is $\ln \left(S_{\text {filter }}\right)$ and $x$ is $1 /$ Temperature. Note that the corresponding temperatures in kelvin are shown on the upper horizontal axis for convenience. 

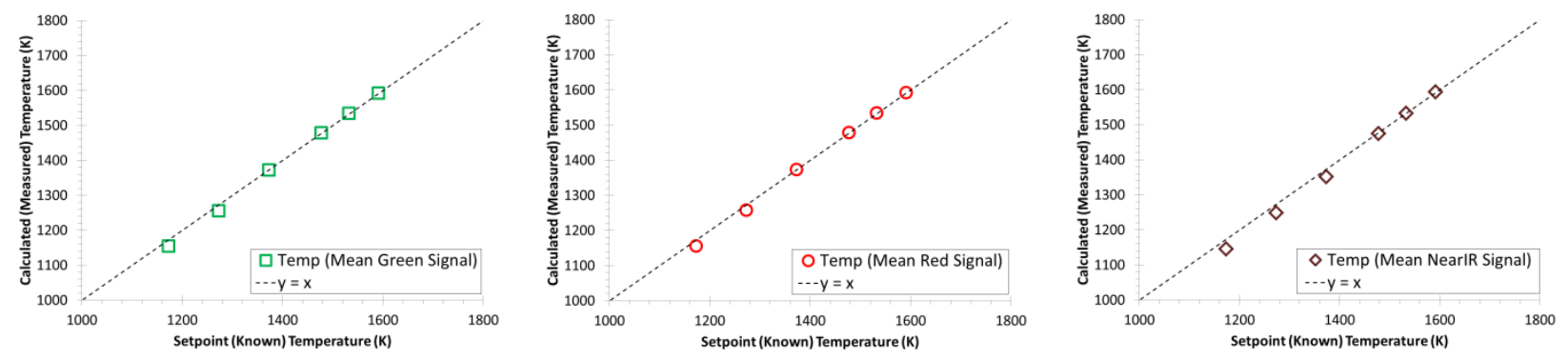

Figure 9. One-Color Pyrometry, Measured vs. Setpoint Temperature, after calibration. Dashed lines represent the line of perfect agreement. The three graphs show results for the (a) Green; (b) Red; and (c) Near IR filter combinations, res pectively.

\section{B. Two-Color Ratio Technique}

The two-color ratio technique has the disadvantage of requiring the acquisition of two images of the same measurement region followed by calculation of the ratio of the signal in those two images. The images can be acquired sequentially, afters witching filters, if the measured object is not changing with time. But if time-resolved measurements are needed, then the images must be acquired simult aneously. This can be accomplished using two separate cameras or using an optical arrangement which results in multiple images on the same sensor. Uncertainties in image registration lead directly to errors in the resulting measurement. This is less of a problem if the measured object is fairly uniform in temperature, but can become the dominant source of error if the measured object has physical structures that are small relative to the resolution of the imaging system and/or has highly localized gradients in temperature.

The primary advantage of the two-color technique is that it does not require re-calibration (or alternatively, an in-situ calibration point) with every change in optical pathway (for example, a change in lens aperture). And as long as the emissivity of the meas ured object is the same at the two filter wavelengths, the effect of emis sivity cancels out when taking the signal ratio. Likewise, if the emissivity is differentbut known at the two wavelengths, the ratio of the emissivity simply becomes an additional constant in the calculation. This allows the calibration performed in one environment to be applied directly to the data taken in another. Furthermore, if three filters are used, a separate temperature measurement can be made using each of the threepossible ratios, resulting in a rough measurement of the uncertainty of the technique.

Figure 10 shows the theoretical signal ratios of various combinations of two filters, revealing the relative sensitivity of each pair. The steeper the slope, the more precise the temperature measurement will be, with the drawback that the dynamic range of measureable temperatures is more limited. This is because for a large intensity ratio, one of the images will eventually approach saturation when the other image is just above detection threshold limits. This limitation can be partially overcome with the addition of neutral den sity filters to the brighter of the two channels and an increase in exposure time.

Calibration of this technique involves comparing the temperature calculated using the curves in Fig. 10 to a known temperature. Figure 11 shows un-calibrated temperature calculations versus setpointtemperatures. From the fits to these data, calibration constants can beextracted. Figure 12 shows the data after calibration and Table 3 lists the calibration constants for each possible filter combination. These constants are derived from the fits in Fig. 10 according to Eq. 8:

$$
\ln \left(\frac{S_{\text {filter } 1}}{s_{\text {filter } 2}}\right)=\frac{a_{\text {filter } 1 / \text { filter } 2}}{T_{o b j}}+b_{\text {filter } 1 / \text { filter } 2}
$$

Rearranging Eq. 8 yields the expression in Eq. 9, which gives the temperature of an object using a measured signal ratio and the calibration constants from Table 3 :

$$
T_{o b j}=\frac{a_{\text {filter } 1 / \text { filter } 2}}{\ln \left(\frac{S_{\text {filter } 1}}{\left.S_{\text {filter } 2}\right)-b_{\text {filter } 1 / \text { filter } 2}}\right.}
$$




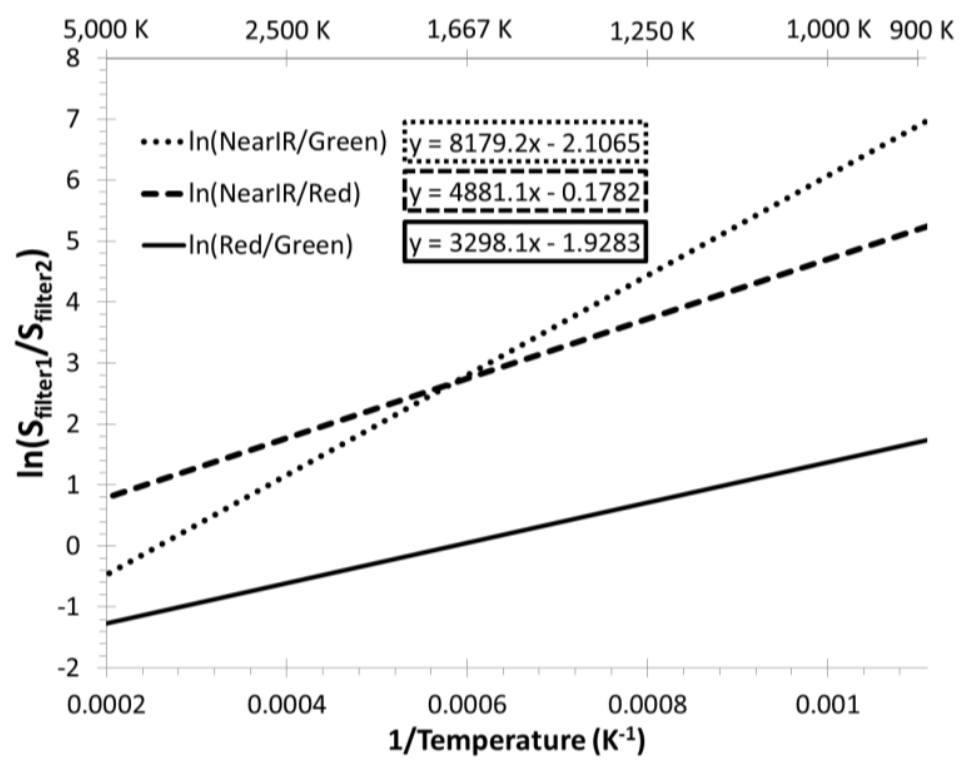

Figure 10. Two-Color Pyrometry Theoretical Curves. The natural logarithm of the signal ratios versus the inverse of temperature is shown for all three filter combinations used in the present study along with equations for each of the three curves, where $y$ is $\ln \left(\mathrm{S}_{\text {filter } 1} / \mathrm{S}_{\text {filter2 }}\right)$ and $\mathrm{x}$ is $1 /$ Temperature. Note that the corresponding temperatures in kelvin are shown on the upper horizontal axis for convenience.
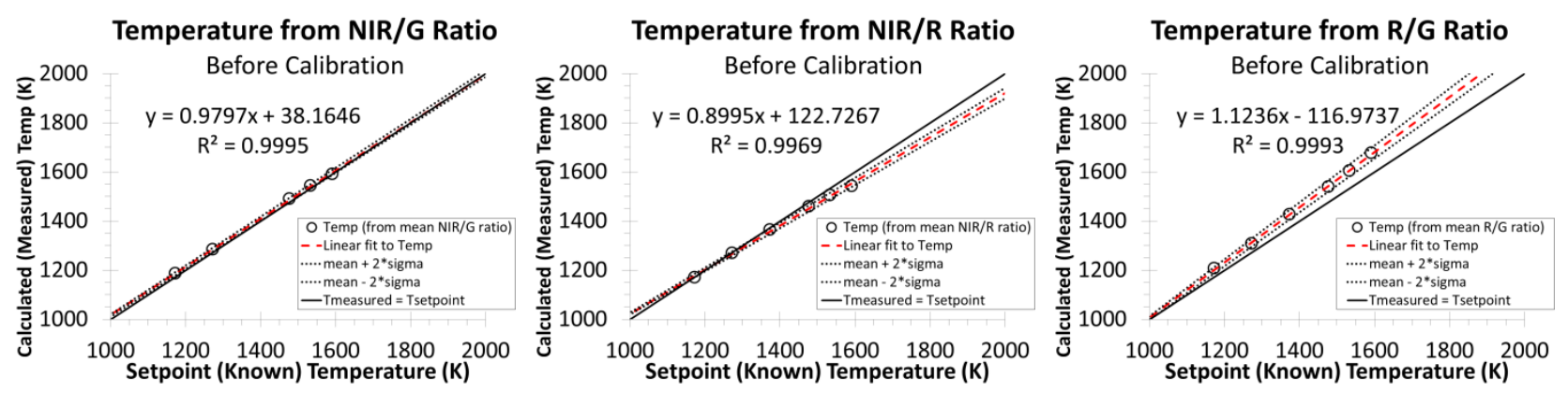

Figure 11. Before Calibration: Two-Color Pyrometry Measured vs. Setpoint Temperature. Solid lines represent the line of perfect agreement. Red dashed lines show fits to the uncalibrated measurements. Black dotted lines indicate two standard deviations away from the fit to the mean. The three filter combinations are (a) Near IR / Green; (b) Near IR / Red; and (c) Red / Green.
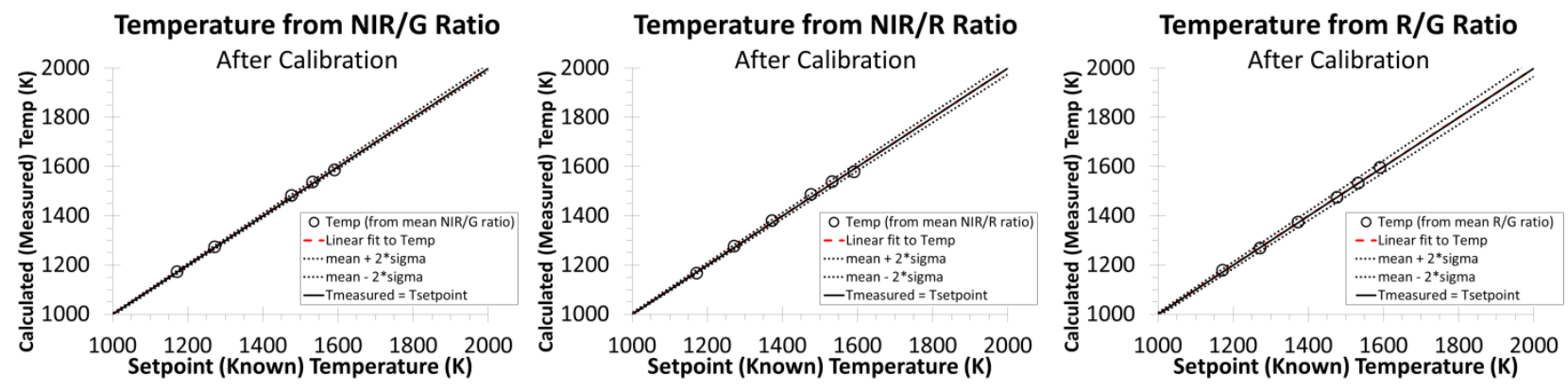

Figure 12. After Calibration: Two-Color Pyrometry Meas ured vs. Setpoint Temperature. Solid lines represent the line of perfect agreement. Red dashed lines are difficult to see since they show fits to the calibrated measurements, which coincide with the line of perfect agreement. Black dotted lines indicate two standard deviations away from the fit to the mean. The three filter combinations are (a) Near IR / Green; (b) Near IR / Red; and (c) Red / Green. 
Table 3. Two-Color Pyrometry Calibrations Constants.

\begin{tabular}{ccc}
\hline Filter Ratio & \multicolumn{2}{c}{$\begin{array}{c}\text { 2-color Pyrometry } \\
\text { constants }\end{array}$} \\
\cline { 2 - 3 } filter1/filter2 & $a$ & $b$ \\
\hline NearlR/Green & 8179.2 & -2.1065 \\
NearIR/Red & 4881.1 & -0.1782 \\
Red/Green & 3298.1 & -1.9283 \\
\hline
\end{tabular}

\section{Conclusion}

A high-speed CMOS-camera based optical pyrometry system has been designed, built, and calibrated for making non-intrusive, high-speed thermal imaging measurements of high-temperature boron during the boron nitride nanotube synthesis process. Spectral emis sion measurements with a spectrometer in the range of relevant temperatures and waveleng ths found boron to be close to an ideal graybody emitter over the range of measured wavelengths, lacking any distinguishable emission or absorption lines or other fine structure. Based upon this observation and the few values of the emis sivity of boron at discrete wavelengths that can be found in the literature, we conclude that theemis sivity of liquid boron is a slowly-varying, nearly-constant function of wavelength over the spectral region of interest (approximately 500-850 $\mathrm{nm}$ ). The narrowband filters used in the present study were chosen specifically because they transmit at wavelengths for which the emis sivity of liquid boron is known from the literature. This observation of near-graybody behavior may permit the use of other filters in the visible and nearvisible range.

An attempt was made to use an 8-bit color Phantom 9.0 high-s peed camera for optical pyrometry, but significant non-linearities and limitations imposed by the dynamic range prompted the authors to use a different camera.

A 12-bit pco.dimax HD CMOS-based camera was found to have good linearity between $5 \%$ and $95 \%$ of its dynamic range, for exposure times of between $30 \mu \mathrm{s}$ and the camera maximum of $40 \mathrm{~ms}$. Using this camera, both one- and two-color pyrometry techniques were investig ated. By imaging the emis sion from Mikron M300 and M360 blackbody radiation calibration sources at various temperatures, calibration constants were determined for all three filters (for the one-color technique) and all three filter ratio combinations (for the two-color technique).

Equations have been provided which allow calculations of temperature from measured signal intensities using the filters employed in this study. For future in-situ meas urements of boron during the BNNT synthesis process, we plan to use the known abrupt emis sivity change at the boron melting/solidification temperature as a calibration point for the one-color technique, and to compare temperatures obtained using that calibration point to those calculated using the emissivity values in Table 1.

Finally, the high-speed imaging capabilities of the chosen camera should enable this system to search for correlations between temperature measurements and other variables, including any dynamic events, involved in the nanotube synthesis process.

\section{Ack nowledgments}

The authors wish to thank David Mercer for his assistance with the blackbody furnaces. This work was supported by the Space Technology Mis sion Directorate, Game Changing Development Program, Nanotechnology Thematic Project (Boron Nitride Nanotube Seedling Project).

\section{References}

\footnotetext{
${ }^{1}$ Michael W. Smith, Kevin C. Jordan, Cheol Park, Jae-Woo Kim, Peter T Lillehei, Roy Crooks and Joycelyn S. Harris on, "Very long single-and few-walled boron nitride nanotubes via the pressurized vapor/condenser method" Nanotechnology, 20 (50) 505604, December 2009. doi:10.1088/0957-4484/20/50/505604

${ }^{2}$ F. Millot, J.C. Rifflet, V. Sarou-Kanian, and G. Wille, "High-Temperature Properties of Liquid Boron from Contactless Techniques," International Journal of Thermophysics, 23 (5), pp. 1185-1195 (September 2002). doi: 10.1023/A:1019836102776

${ }^{3}$ Shankar Krishnan, PaulC. Nordine, J.K. Richard Weber and Robert A. Schiffman, "Optical Properties and Melting Point of Pure Boron,” High Temp. Sci., 31, pp. 45-57 (1991).
} 
${ }^{4}$ L.A. Koval, A.V. Florko, and Ya. I. Vovchuk, "Emissivity Characteristics of Boron and Boric Oxide at High Temperatures," Combustion, Explosion, and Shock Waves, 46 (2) pp. 178-182 (2010). Trans. from Fizika Goreniyai Vzryva, 46 (2) pp. 68-73, March-April, 2010. doi: 10.1007/s 10573-010-0027-3

${ }^{5}$ S. M. Sze and Kwok K. Ng, Physics of Semiconductor Devices, $3^{\text {rd }}$ ed., John Wiley and Sons, N.Y., 1981, Chap. 13 , p.665

${ }^{6}$ Arnaud Darmont, "Spectral Res ponse of Silicon Image Sensors," Aphes a White Paper, April 2009, available via URL: http://www.aphesa.com/documents.php [retrieved 8 May 2014]

${ }^{7}$ David H. Terry, MichaelE. Thomas, Milton J. Linevsky, and Daniel T. Prendergast, "Imaging Pyrometry of LaserHeated Sapphire," Johns Hopkins APL Technical Digest 20 (2) pp. 162-169 (1999). available via URL: http://techdigest.jhuapl.edu/TD/td2002/Terry.pdf

${ }^{8}$ Paul M. Danehy, Drew V. Hires, Craig T. Johansen, Brett F. Bathel, Stephen B. Jones, Jeffrey G. Gragg, and Scott C. Splinter, "Quantitative Spectral Radiance Measurements in the HYMETS Arc Jet", 50th AIAA Aerospace Sciences Meeting, Nashville, TN, AIAA Paper 2012-856, 9-12 January 2012.

${ }^{9}$ Data sheet for Mikron M300, available via URL: http://www.transcat.com/PDF/blackbody.pdf [retrieved 8 May 2014]

${ }^{10}$ Data sheet for Mikron M360, available via URL: http://www.transcat.com/PDF/m335 mikron.pdf [retrieved 8 May 2014]

${ }^{11}$ Vladimir L. Solozhenko and Oleksandr O. Kurakevych, "Equilibriump-T Phase Diagram of Boron: Experimental Study and Thermodynamic Analysis," Scientific Reports 3, Article number: 2351 (2013). doi:10.1038/srep02351

${ }^{12}$ Tairan Fu, Xiaofang Cheng, and Zangjian Yang, "Theoretical evaluation of measurement uncertainties of two color pyrometry applied to optical diagnostics," Applied Optics 47 (32) pp. 6112-6123 (10 November 2008). doi: 10.1364/AO.47.006112

${ }^{13}$ John M. Densmore, Matthew M. Biss, Kevin L. McNesby, and Barrie E. Homan, "High-Speed digital color imaging pyrometry," Applied Optics, 50 (17), pp. 2659-2665 (10 June 2011). doi:10.1364/AO.50.002659 